(2) Open Access Full Text Article

\title{
Expression of pyruvate kinase $M 2$ in human bladder cancer and its correlation with clinical parameters and prognosis
}

This article was published in the following Dove Press journal:

OncoTargets and Therapy

\author{
Changkun Huangl,* \\ Zhichao Huangl,* \\ Peiming $\mathrm{Bai}^{2}$ \\ Guangcheng Luo ${ }^{2}$ \\ Xiaokun Zhao' \\ Xinjun Wang ${ }^{2}$
}

'Department of Urology, The Second Xiangya Hospital, Central South University, Changsha, People's Republic of China; ${ }^{2}$ Department of Urology, Zhongshan Hospital, Xiamen University, Xiamen, People's Republic of China

*These authors contributed equally to this work
Correspondence: Xinjun Wang Department of Urology, Zhongshan Hospital, Xiamen University, No 20I, South Hubin Road, Siming District, Xiamen, Fujian 361004, People's

Republic of China

$\mathrm{Tel}+86 \quad 8030239818$

Fax +865922212328

Emailwxj@xmu.edu.cn
Background: Pyruvate kinase M2 (PKM2) is a key regulator of the Warburg effect and has critical functions in glycolysis, contributing to the Warburg effect, tumor growth, angiogenesis, cell division, metastasis, and apoptosis. The high expression of PKM2 in various solid tumors renders it a potential biomarker of tumorigenesis and tumor invasion, but the expression and role of PKM2 in bladder cancer have not been studied extensively.

Patients and methods: Western blot and immunohistochemistry (IHC) were used to measure the expression of PKM2, and quantitative real-time polymerase chain reaction (PCR) was performed to determine PKM2 mRNA levels. The relationships between PKM2 expression and clinicopathological parameters and prognosis were analyzed using the Kaplan-Meier plots and a Cox proportional hazards regression model.

Results: Compared with paired adjacent normal bladder tissues, PKM2 mRNA and protein levels were found to be higher in urothelial carcinoma of the bladder (UCB) samples by realtime PCR and Western blot. By IHC, high expression of PKM2 was seen in 117 of 215 UCBs (54.4\%) and in eight of 90 adjacent normal bladder tissues (8.9\%). The expression of PKM2 was significantly associated with grade, stage, and lymph node status $(P<0.001)$. In the univariate survival analysis, a significant association between PKM2 expression and shorter patient survival was observed $(P<0.001)$. In different subsets of UCB patients, we found that PKM2 expression was a prognostic factor in patients with G2 $(P=0.009), \mathrm{G} 3(P<0.001), \mathrm{pTa} / \mathrm{pT}$ is $(P=0.006)$, pT1, pT2-4, and $\mathrm{pN}^{-}$disease $(P<0.001)$. Importantly, PKM2 expression $(P=0.003)$, with tumor histological grade $(P<0.001)$, pT $(P<0.001)$, and $\mathrm{pN}$ status $(P=0.005)$, was a significant independent prognostic parameter in the multivariate analysis.

Conclusion: PKM2 protein and mRNA are upregulated in UCBs and may serve as molecular markers for a poor prognosis in patients with UCB.

Keywords: bladder, urothelial carcinoma, PKM2, prognosis, IHC

\section{Introduction}

Bladder cancer $(\mathrm{BCa})$ is one of the most common malignancies of the urinary tract, of which urothelial carcinoma of the bladder (UCB) is the most frequent histological subtype. Over the past several decades, the incidence of $\mathrm{BCa}$ has increased; the incidence of $\mathrm{BCa}$ in males and females in developing countries was 16.9/100,000 and $3.7 / 100,000$, respectively, in $2012 .{ }^{1}$ Well-recognized tools for predicting the prognosis include clinical staging and pathological grading, while it is difficult to explain why patients with $\mathrm{BCa}$ of the same stage and grade have different prognoses. There are few molecular markers that predict the prognosis of $\mathrm{BCa}$. 
Increased conversion of glucose to lactate is a key feature of many cancer cells, which promotes rapid growth. The type $\mathrm{L}$ and $\mathrm{R}$ pyruvate kinase isoenzymes are encoded by the same gene but are under the control of different promoters. ${ }^{2-8}$ The type M1 and M2 pyruvate kinase isoenzymes are different splice products from the same mRNA transcript, resulting in a difference of 21 amino acids. ${ }^{9,10}$ Pyruvate kinase M2 (PKM2) has been proposed to be a candidate oncogene that is upregulated in several cancers, including renal cell carcinoma (RCC), ${ }^{11,12}$ ovarian cancer, ${ }^{13,14}$ breast cancer,,${ }^{15,16}$ and lung cancer. ${ }^{17}$

In this study, quantitative real-time polymerase chain reaction (PCR), Western blot, and immunohistochemistry (IHC) were used to detect the expression of PKM2 in tumor and adjacent tissues. In addition, the relationship between the expression of PKM2 and clinicopathological parameters was analyzed. Kaplan-Meier plots and a Cox proportional hazards regression model were used for the univariate and multivariate survival analyses.

\section{Patients and methods}

\section{Patients and samples}

For the qRT-PCR and Western blot analysis, 10 paired fresh UCB tissues and normal bladder tissues adjacent to the tumor (defined as $>2.0 \mathrm{~cm}$ from the tumor edge) were collected from consecutive patients with UCB who underwent surgery in the Department of Urology, The Second Xiangya Hospital, Central South University (Changsha, People's Republic of China) from January 2012 to November 2012. In addition, 215 formalin-fixed, paraffin-embedded UCB tissues, diagnosed by the Departments of Pathology and Urology, The Second Xiangya Hospital, Central South University (Changsha, People's Republic of China), were retrieved. We selected cases according to the typical pathological diagnosis of UCB, without chemotherapy or radiotherapy prior to surgery.

Each patient was staged according to the 2009 The Union for International Cancer Control staging system. ${ }^{18}$ In brief, the 215 patients included 145 males and 70 females, with a median age of 62 years (range: $20-88$ ). The median follow-up time for overall survival (OS) was 62 months. Ninety-nine patients underwent radical cystectomy and 116 underwent transurethral resection of the bladder tumor. The clinicopathological features of the 215 patients are summarized in Table 1. Written informed consent was obtained from the patients for use of their tissue specimens and medical data, and the study protocol was approved by the Institutional Research Ethics Committee of Second Xiangya Hospital, Central South University.
Table I Correlation between PKM2 expression and clinicopathological characteristics of UCB patients

\begin{tabular}{|c|c|c|c|c|}
\hline \multirow[t]{2}{*}{ Characteristics } & \multicolumn{3}{|c|}{ PKM2 protein } & \multirow[t]{2}{*}{$P$-value ${ }^{a}$} \\
\hline & $\begin{array}{l}\text { Total } \\
\text { cases }\end{array}$ & Low & High & \\
\hline Age (years) & & & & 0.273 \\
\hline$\leq 65^{b}$ & 113 & 56 & 57 & \\
\hline$>65$ & 102 & 42 & 60 & \\
\hline Gender & & & & 0.465 \\
\hline Male & 145 & 69 & 76 & \\
\hline Female & 70 & 29 & 41 & \\
\hline Histological grade & & & & $<0.001$ \\
\hline GI & 71 & 55 & 16 & \\
\hline $\mathrm{G} 2$ & 62 & 29 & 33 & \\
\hline G3 & 82 & 14 & 68 & \\
\hline PT classification & & & & $<0.001$ \\
\hline $\mathrm{pTa} / \mathrm{p} T$ is & 61 & 42 & 19 & \\
\hline PTI & 61 & 33 & 28 & \\
\hline pT2-4 & 93 & 23 & 70 & \\
\hline $\mathrm{pN}$ classification & & & & $<0.001$ \\
\hline $\mathrm{pN}^{-}$ & 169 & 95 & 74 & \\
\hline $\mathrm{pN}^{+}$ & 46 & 3 & 43 & \\
\hline Tumor size (cm) & & & & 0.336 \\
\hline$\leq 3^{c}$ & 119 & 58 & 61 & \\
\hline$>3$ & 96 & 40 & 56 & \\
\hline Tumor multiplicity & & & & 0.132 \\
\hline Unifocal & 110 & 56 & 54 & \\
\hline Multifocal & 105 & 42 & 63 & \\
\hline
\end{tabular}

Notes: ${ }^{2} \mathrm{Chi}-$ square test. ${ }^{b}$ Median age. ${ }^{\mathrm{M}}$ Mean size.

Abbreviations: PKM2, pyruvate kinase M2; UCB, urothelial carcinoma of the bladder.

\section{real-time PCR analysis}

Total RNA was isolated using TRizol reagent (Thermo Fisher Scientific, Waltham, MA, USA). RNA was reverse-transcribed to cDNA using the RevertAid ${ }^{\mathrm{TM}} \mathrm{H}$ Minus First Strand cDNA Synthesis Kit (Thermo Fisher Scientific) according to the manufacturer's instructions. The qRT-PCR was performed using SYBR Green PCR Master Mix (Applied Biosystems, Foster City, CA, USA) in a total reaction volume of $25 \mu \mathrm{L}$ on an ABI PRISM 7900HT Sequence Detection System (Applied Biosystems) as follows: denaturation for 5 minutes at $95^{\circ} \mathrm{C}$; 40 cycles of denaturation for 20 seconds at $94^{\circ} \mathrm{C}$, annealing for 20 seconds at $61^{\circ} \mathrm{C}$, extension for 20 seconds at $72^{\circ} \mathrm{C}$; and a final extension for 5 minutes at $72^{\circ} \mathrm{C}$. The specificity of the amplified products was determined by melting curve analysis. The relative expression levels of mRNA were calculated by the $2^{-\Delta \Delta \mathrm{Ct}}$ method and expressed as relative quantification values, normalized to $\beta$-actin. Experiments were repeated in triplicate. The primers used in this study were as follows: PKM2 forward (5'-ATTATTTGAGGAACTCCGCCGCCT-3') and reverse (5'-ATTCCGGGTCACAGCAATGATGG-3); $\beta$-actin forward (5'-CATTAAGGAGAAGCTGTGCT-3') and reverse (5'-GTTGAAGGTAGTTTCGTGGA-3'). 


\section{Western blot}

Tissue proteins were lysed in radioimmunoprecipitation assay buffer, and the total protein concentration was determined by the Bradford method: $20 \mu \mathrm{g}$ of protein was loaded onto $8 \%$ denaturing Sodium dodecyl sulfate polyacrylamide gel electrophoresis for electrophoresis. The blots were probed with the primary antibodies overnight at $4^{\circ} \mathrm{C}$, followed by incubation with the appropriate secondary antibodies at room temperature for 1 hour. PKM2 rabbit monoclonal anti-PKM2 (1:1,000; Cell Signaling Technology, Danvers, MA, USA) and goat anti-rabbit (1:1,000; Santa Cruz Biotechnology, Dallas, TX, CA) antibodies were used to detect PKM2 protein.

\section{Immunohistochemical staining}

Deparaffinized and rehydrated tissue sections were pretreated with $3 \%$ peroxidase in methanol for 10 minutes at room temperature. Antigen retrieval was performed by boiling the sections in citrate buffer $(\mathrm{pH} 6.0)$ for 30 minutes. After preincubation with the blocking buffer (10\% normal goat serum in PBS) for 1 hour at room temperature, the sections were incubated with PKM2 (diluted 1:200) (Cat:4053; Cell Signaling Technology) overnight at $4^{\circ} \mathrm{C}$, followed by incubation with the secondary antibody (KIT-5010, MaxVision $^{\mathrm{TM}}$ ) for 1 hour. PKM2 signals were visualized by $3,3^{\prime}$-diaminobenzidine staining.

Each section was evaluated independently by two pathologists. Each slide was observed in five fields of view, with each field of view magnified 400 times to observe 100 cells. The expression of PKM2 was scored according to staining intensity and extent. The absence of reactivity was considered to be negative. According to the percentage of positively stained area, the sections were given scores of $0(0 \%), 1(1 \%-25 \%), 2(26 \%-50 \%), 3(51 \%-75 \%)$, and 4 points ( $76 \%-100 \%)$. Also, according to the degree of staining, the samples received a score of 0 (negative: no staining), 1 (weak positive: light yellow), 2 (medium positive: brown), or 3 points (strong positive: tan). Both scores were added to yield the final score ( $0-7$ points). Final scores $>3$ indicated high expression and those $\leq 3$ indicated low expression. ${ }^{19}$

\section{Statistical analyses}

All statistical analyses were performed with SPSS 17.0. The association of PKM2 expression with clinicopathological features of the $\mathrm{BCa}$ patients was assessed using the $\chi^{2}$ test. Survival was analyzed by the Kaplan-Meier method. Logrank test was used to compare different survival curves. Univariate and multivariate survival analyses were performed using the Cox proportional hazards regression model.
Differences were considered to be significant if the $P$-value by the two-tailed test was $<0.05$.

\section{Results \\ Expression of PKM2 mRNA and protein in paired UCB and adjacent tissues}

The relative expression of PKM2 mRNA in UCB and adjacent normal tissues was $5.878 \pm 1.346$ and $3.683 \pm 0.7561$, respectively $(P<0.001)$ (Figure $1 \mathrm{~J})$. The relative expression of $\mathrm{PKM} 2$ protein in $\mathrm{BCa}$ tissues was $1.347 \pm 0.576$, which was significantly higher than in adjacent normal tissues $(0.642 \pm 0.255)(P<0.001)$ (Figures $1 \mathrm{I}-1 \mathrm{~K})$.

\section{Expression of PKM2 in UCBs by IHC}

We further examined the expression of PKM2 protein in 215 paraffin-embedded UCB tissues and 90 matched normal tissues by IHC. PKM2 expression was substantially upregulated in tumor tissues compared with normal tissues. Of all UCB tissues, 54.4\% (117/215) showed high expression of PKM2, compared with 8.9\% (8/90) of controls. Positivity for PKM2 occurred primarily in the cytoplasm (Figure 1A-H and Table 2).

\section{Correlation between PKM2 expression and clinicopathological features}

The relationship between the clinicopathological characteristics of UCB patients and PKM2 levels was analyzed. Only three factors $-\mathrm{T}$ classification $(P<0.001)$, histological grade $(P<0.001)$, and $\mathrm{N}$ classification $(P<0.001)$ - were significantly associated with high expression of PKM2 (Table 1).

\section{Relationship between clinicopathological features, PKM2 expression, and UCB patient survival: univariate survival analysis}

To determine the clinical significance of PKM2 expression in UCB patients, survival curves were created, and we found that overexpression of PKM2 correlated with a poor prognosis in the UCB patients in this study. Both the OS and disease-free survival (DFS) rates of UCB patients with low expression of PKM2 were higher than those of high expressors (Figures 2 and 3; both $P<0.001$ ). The univariate Cox proportional hazards regression model demonstrated that patients with high PKM2 expression had a greater risk of death than those with low expression (hazard ratio $[\mathrm{HR}]=0.132,95 \%$ confidence interval $[\mathrm{CI}]: 0.073-0.239$, $P<0.001$ ) (Table 2).

Furthermore, PKM2 expression was demonstrated to be a prognostic factor for OS in UCB patients with $\mathrm{G} 2(P=0.009)$, 

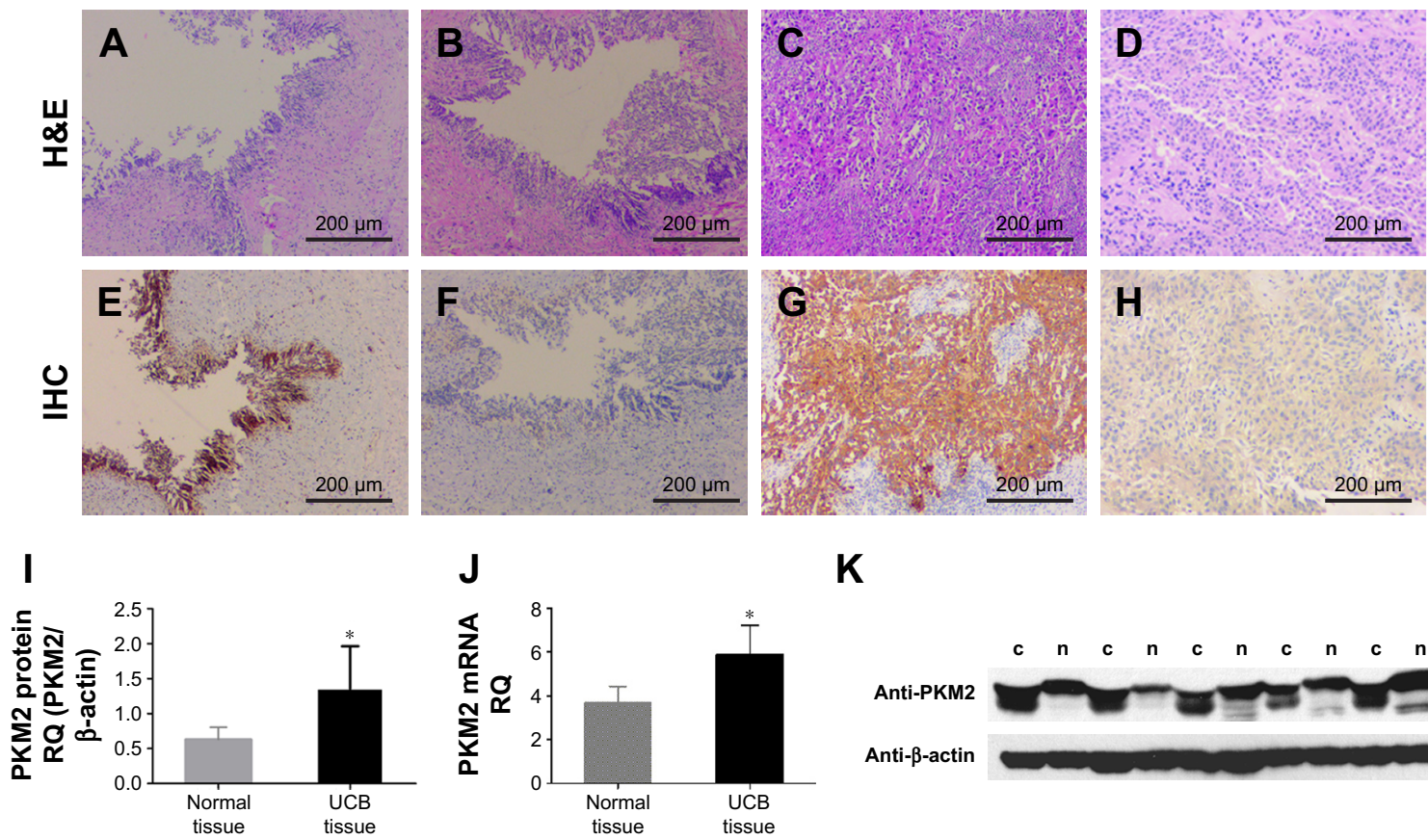

K

Figure I Expression of PKM2 in UCBs and adjacent normal bladder tissues.

Notes: (A-D) Representative PKM2 and H\&E stains are shown. Positivity for PKM2 was observed primarily in the cytoplasm. (A and B) Adjacent normal bladder tissues observed by H\&E staining $(\times 40)$. (C and D) Bladder cancer tissues observed by H\&E staining $(\times 40)$. (E) High and (F) low expression of PKM2 in adjacent normal bladder tissues observed by IHC $(\times 40)$. (G) High and $(\mathbf{H})$ low expression of PKM2 in bladder cancer tissues observed by IHC $(\times 40)$. (I and $\mathbf{K})$ Relative expression of PKM2 protein detected by Western blot in 10 UCB cases. Expression levels were normalized to $\beta$-actin. (J) Relative expression of PKM2 mRNA detected by real-time-PCR in IO UCB cases. *P $\angle 0.001$. Abbreviations: PKM2, pyruvate kinase M2; UCB, urothelial carcinoma of the bladder; H\&E, hematoxylin and eosin; IHC, immunohistochemistry; PCR, polymerase chain reaction; RQ, relative quantification; c, bladder cancer tissues; $\mathrm{n}$, normal bladder tissues.

Table 2 Univariate analysis of different prognostic factors in 215 patients with $U C B$

\begin{tabular}{|c|c|c|c|}
\hline Characteristics & $\begin{array}{l}\text { Total } \\
\text { cases }\end{array}$ & HR (95\% Cl) & $P$-value \\
\hline Age (years) & & & 0.802 \\
\hline$\leq 65$ & 113 & 1 & \\
\hline$>65$ & 102 & $0.948(0.626-1.436)$ & \\
\hline Gender & & & 0.06 \\
\hline Male & 145 & 1 & \\
\hline Female & 70 & $0.559(0.366-0.854)$ & \\
\hline Histological grade & & & $<0.001$ \\
\hline GI & 71 & I & \\
\hline $\mathrm{G} 2$ & 62 & $0.032(0.010-0.101)$ & \\
\hline G3 & 82 & $0.348(0.243-0.607$ & \\
\hline PT classification & & & $<0.001$ \\
\hline $\mathrm{pTa} / \mathrm{p} T$ is & 61 & 1 & \\
\hline PTI & 61 & $0.032(0.010-0.103)$ & \\
\hline PT2-4 & 93 & $0.240(0.14 \mid-0.409)$ & \\
\hline $\mathrm{pN}$ classification & & & $<0.001$ \\
\hline $\mathrm{pN}^{-}$ & 169 & 1 & \\
\hline $\mathrm{pN}^{+}$ & 46 & $0.130(0.083-0.203)$ & \\
\hline Tumor size $(\mathrm{cm})$ & & & 0.155 \\
\hline$\leq 3$ & 119 & 1 & \\
\hline$>3$ & 96 & $0.74 \mid(0.489-I .122)$ & \\
\hline Tumor multiplicity & & & 0.095 \\
\hline Unifocal & 110 & I & \\
\hline Multifocal & 105 & $0.937(0.619-1.417)$ & \\
\hline PKM2 expression & & & $<0.001$ \\
\hline Low & 98 & 1 & \\
\hline High & 117 & $0.132(0.073-0.239)$ & \\
\hline
\end{tabular}

Abbreviations: UCB, urothelial carcinoma of the bladder; HR, hazard ratio; $\mathrm{Cl}$, confidence interval; PKM2, pyruvate kinase M2.
G3 $(P=0.001), \mathrm{pTa} / \mathrm{p}$ Tis $(P=0.006), \mathrm{pT} 1(P<0.001), \mathrm{pT} 2-4$ $(P<0.001), \mathrm{N}^{-}(P<0.001)$, and $\mathrm{pN}^{+}$disease $(P<0.027)$ (Figure 2). The univariate analysis indicated that OS was related to histological grade $(P<0.001)$, T classification $(P<0.001), \mathrm{N}$ classification $(P<0.001)$, and expression of PKM2 $(P<0.001)$.

PKM2 expression was also confirmed to be a prognostic factor for DFS in UCB patients with G1 $(P<0.001)$, G2 $(P=0.006), \mathrm{G} 3(P<0.001), \mathrm{pTa} / \mathrm{pTis}(P<0.001)$, pT1 $(P<0.001), \mathrm{pT} 2-4(P<0.001)$, and $\mathrm{pN}^{-}$disease $(P<0.001)$ (Figure 3).

\section{Multivariate analysis using Cox proportional hazards regression model for independent prognostic factors}

In the multivariate analysis, when controlling for the effects of standard clinicopathological parameters, the expression of PKM2 was found to be an independent prognostic factor of OS (HR=3.197, 95\% CI: 1.682-6.076, $P<0.001)$ (Table 3). Moreover, we also confirmed histological grade $(\mathrm{HR}=2.446$, 95\% CI: $1.671-3.579, P<0.001)$, T classification $(\mathrm{HR}=2.867$, 95\% CI: $1.901-4.323, P<0.001)$, and $\mathrm{N}$ classification (HR=2.013, 95\% CI: $1.236-3.279, P=0.005)$ to be independent predictors of OS in patients with UCB (Table 3). 
A

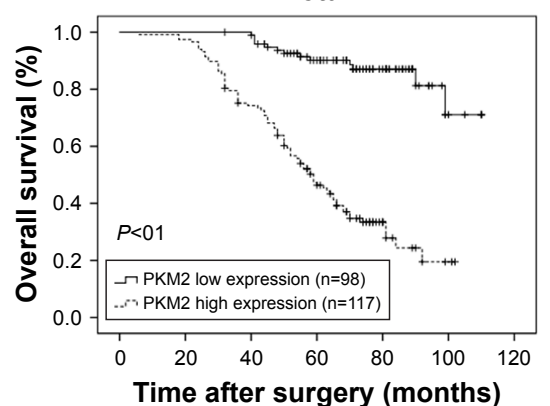

D

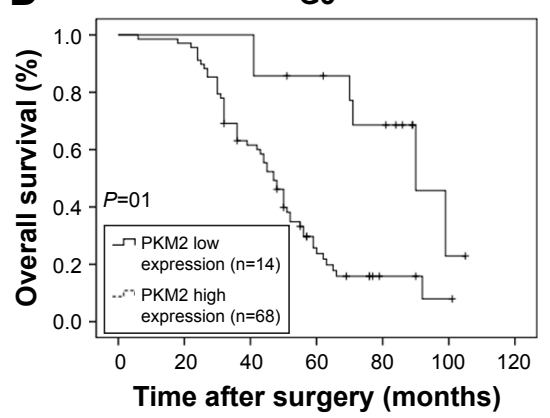

G

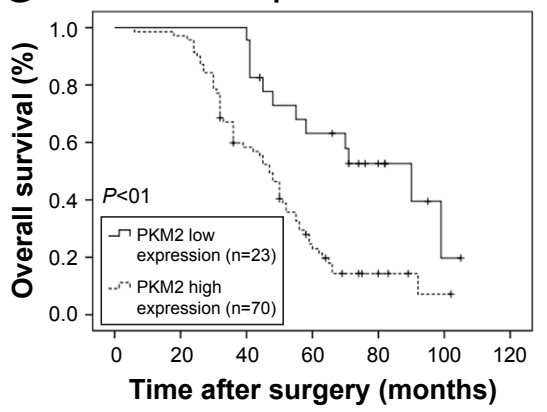

B

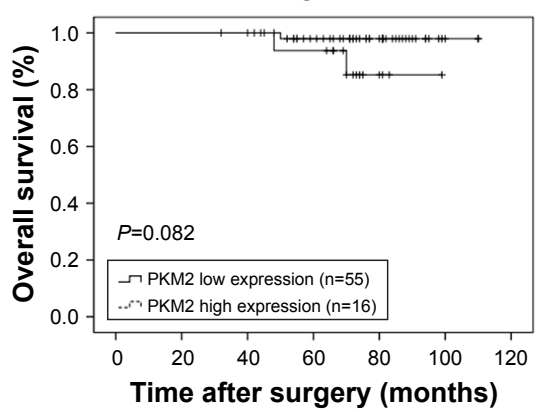

E

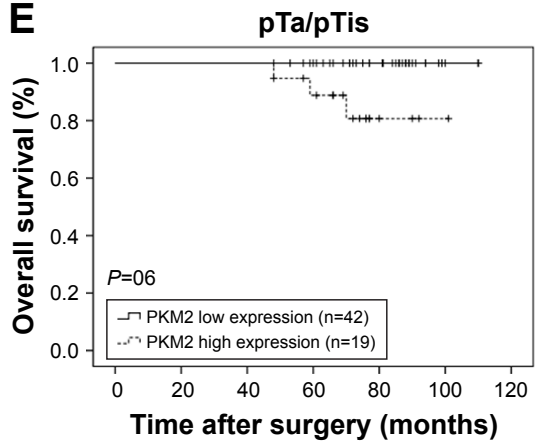

H

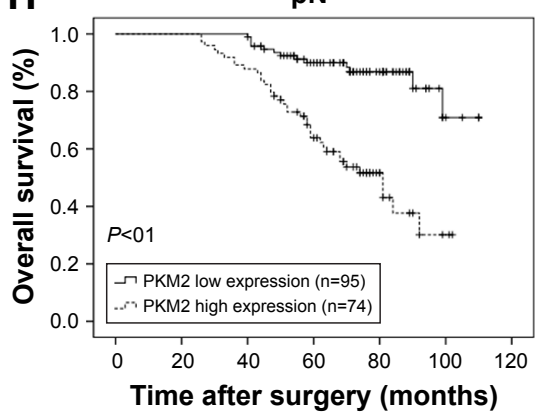

C

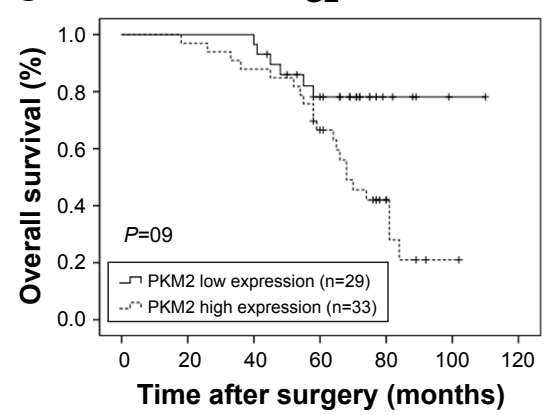

F

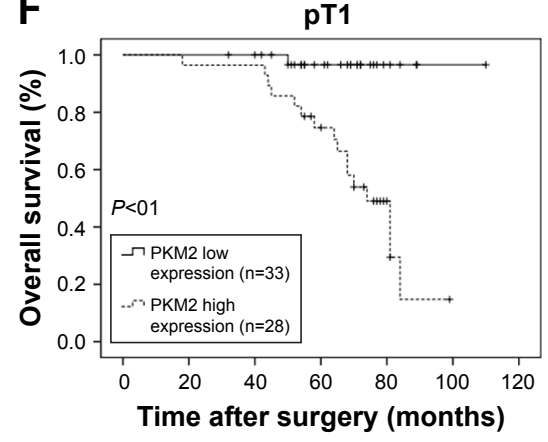

I

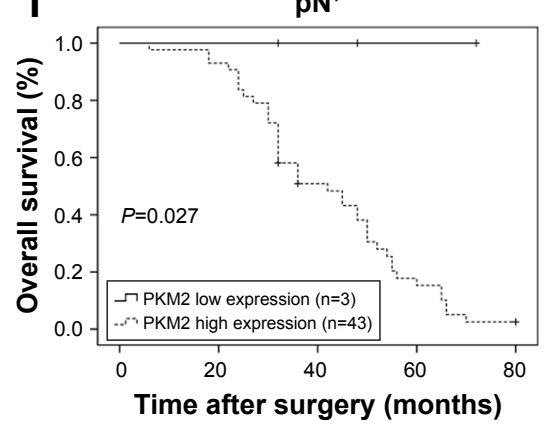

Figure 2 Kaplan-Meier survival analysis of PKM2 expression in patients with UCB (log-rank test).

Notes: Patients with high expression of PKM2 were significantly associated with poorer overall survival $(P<0.001)$. (A) Total, the probability of survival of all patients with UCB: low expression (solid line), $n=98$; high expression (dashed line), $n=\mid$ I7. (B) $\mathrm{GI}$, the probability of survival of $\mathrm{GI}$ patients with UCB: low expression (solid line), $\mathrm{n}=55$; high expression (dashed line), $n=16$. (C) G2, the probability of survival of G2 patients with UCB: low expression (solid line), $n=29$; high expression (dashed line), $n=33$. (D) G3, the probability of survival of G3 patients with UCB: low expression (solid line), n=l4; high expression (dashed line), n=68. (E) pTa/pTis, probability of survival of pTa/pTis patients with UCB: low expression (solid line), $n=42$; high expression (dashed line), $n=19$. (F) pTI, the probability of survival of pTI patients with UCB: low expression (solid line), $n=33$; high expression (dashed line), $n=28$. (G) $p T 2-4$, probability of survival of $p T 2-4$ patients with UCB: low expression (solid line), $n=23$; high expression (dashed line), $\mathrm{n}=70$. (H) $\mathrm{pN}^{-}$, the probability of survival of $\mathrm{pN}^{-}$patients with $\mathrm{UCB}$ : low expression (solid line), $\mathrm{n}=95$; high expression (dashed line), $\mathrm{n}=74$. (I) $\mathrm{p} \mathrm{N}^{+}$, probability of survival of $\mathrm{pN}^{+}$patients with UCB: low expression (solid line), $\mathrm{n}=3$; high expression (dashed line), $\mathrm{n}=43$.

Abbreviations: PKM2, pyruvate kinase M2; UCB, urothelial carcinoma of the bladder.

\section{Discussion}

PKM2, an isoform of pyruvate kinase, is abundantly expressed in rapidly proliferating tissues, such as embryonic tissues and tumors, and in differentiated tissues. ${ }^{20}$ The metabolism of many tumor cells is characterized by elevated glucose uptake but a lower rate of oxidative phosphorylation. This persistence of high lactate production by tumors in the presence of oxygen, known as aerobic glycolysis, was first noted by Warburg $>75$ years ago. ${ }^{21}$ Compared with normal cells, tumor cells give priority to anaerobic oxidation, even under sufficient oxygen, resulting in greater lactic acid production, which is mediated by PKM2 in many tumor cells. PKM2 is necessary for the shift in cellular metabolism to aerobic glycolysis, which promotes tumorigenesis, cell division, apoptosis, and metastasis. ${ }^{22,23}$ Several types of tumor cells have been shown to express the embryonic M2 isoform of pyruvate kinase exclusively. ${ }^{24,25}$

Overexpression of PKM2 is closely associated with many human cancers, such as RCC, ${ }^{11,26}$ ovarian cancer, ${ }^{13,14}$ breast cancer, ${ }^{15}$ and lung cancer. ${ }^{17}$ Bluemlein et al found that PKM2 is highly expressed in $\mathrm{BCa}$ by mass spectrometry. ${ }^{27}$ In the current study, the expression of PKM2 was examined in $\mathrm{BCa}$ tissues. Collectively, these findings suggest a potential oncogenic role of PKM2 in BCa. 
A
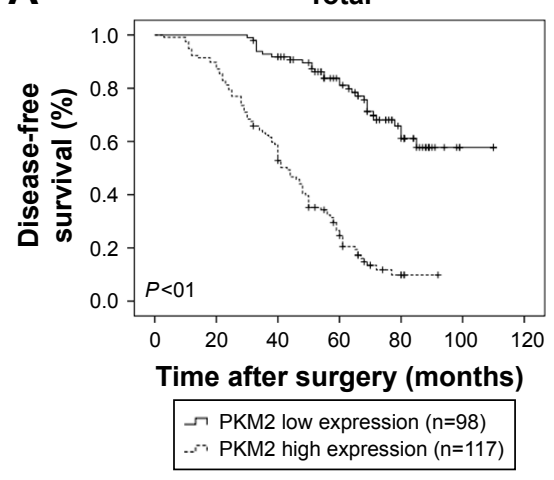

D
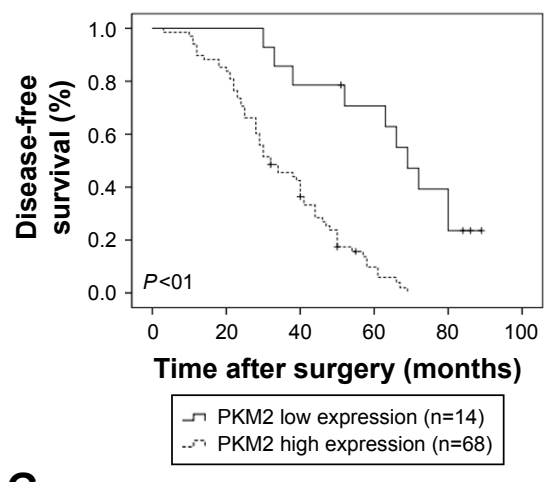

G

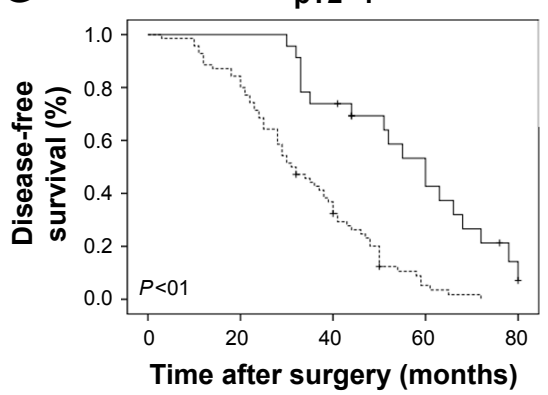

$\neg$ PKM2 low expression $(n=23)$

$\cdots$ PKM2 high expression $(\mathrm{n}=70)$
B
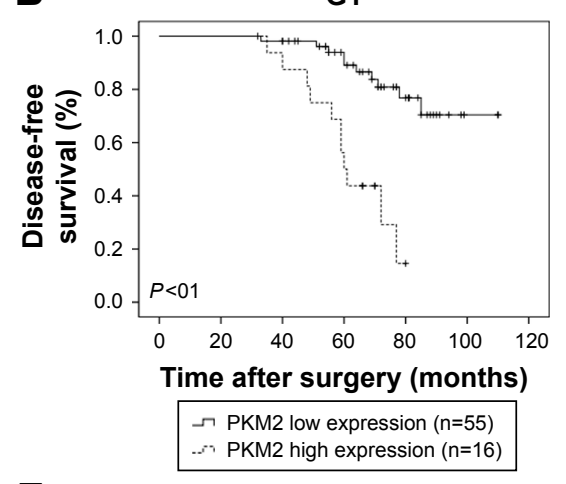

E
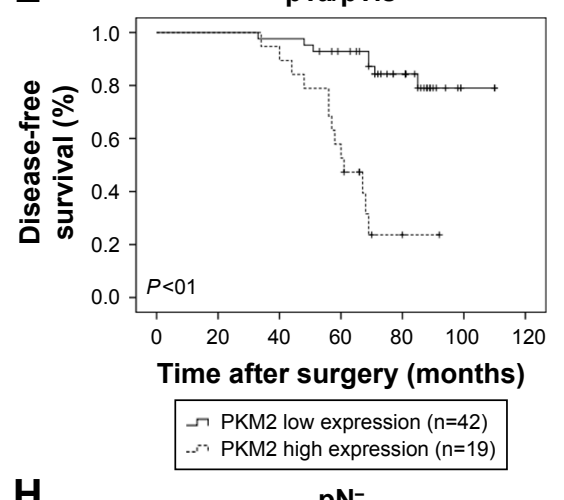

H

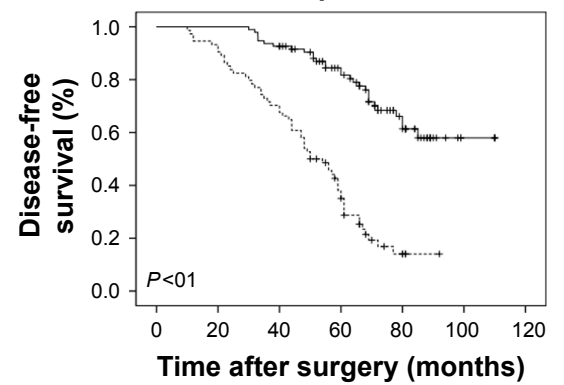

$\rightarrow$ PKM2 low expression $(n=95)$

$\because$ PKM2 high expression $(n=74)$
C $\quad$ G2

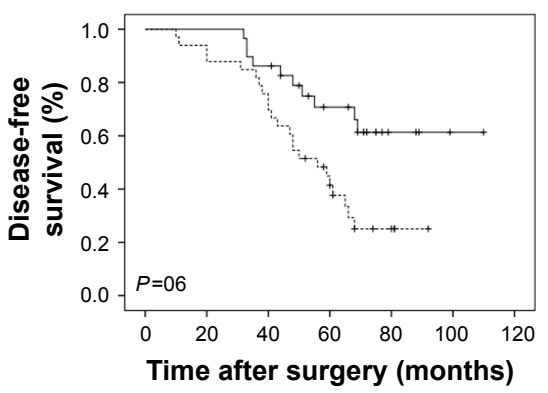

$\sim$ PKM2 low expression $(n=29)$ .. PKM2 high expression $(n=33)$

F

pT1
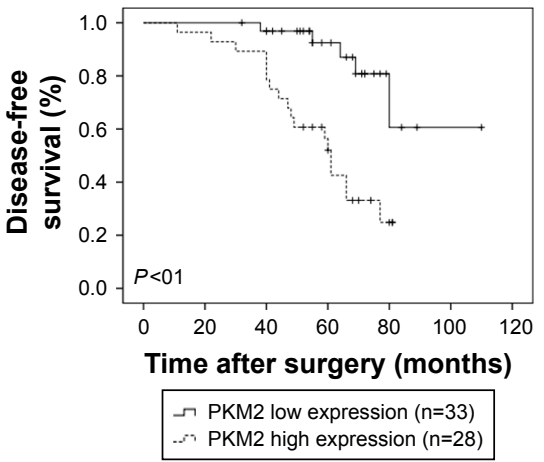

I

$\mathrm{pN}^{+}$

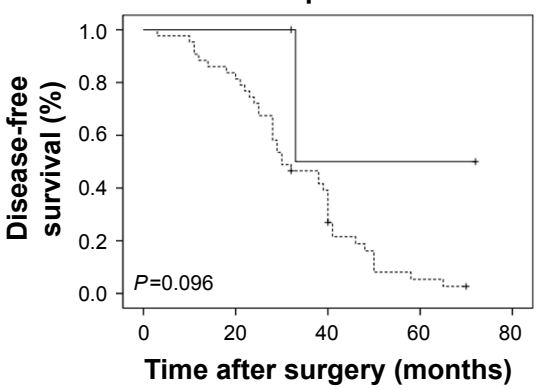

Time after surgery (months)

$\neg$ PKM2 low expression $(n=3)$

Figure 3 Kaplan-Meier disease-free survival analysis of PKM2 expression in patients with UCB (log-rank test).

Note: Patients with high expression of PKM2 were significantly associated with poorer disease-free survival $(P<0.00 \mathrm{I})$. (A) Total, the probability of survival of all patients with UCB: low expression (solid line), $n=98$; high expression (dashed line), $n=I$ I7. (B) GI, the probability of survival of GI patients with UCB: low expression (solid line), $\mathrm{n}=55$; high expression (dashed line), $\mathrm{n}=16$. (C) $\mathrm{G} 2$, the probability of survival of $\mathrm{G} 2$ patients with UCB: low expression (solid line), $\mathrm{n}=29$; high expression (dashed line), $n=33$. (D) G3, the probability of survival of G3 patients with UCB: low expression (solid line), $n=\mid 4$; high expression (dashed line), $n=68$. (E) $p T a / p T$ is, probability of survival of $\mathrm{pTa} / \mathrm{pT}$ is patients with UCB: low expression (solid line), $n=42$; high expression (dashed line), $n=19$. (F) pTI, the probability of survival of $\mathrm{pTI}$ patients with UCB: low expression (solid line), n=33; high expression (dashed line), n=28. (G) pT2-4, probability of survival of pT2-4 patients with UCB: low expression (solid line), $n=23$; high expression (dashed line), $\mathrm{n}=70$. $(\mathbf{H}) \mathrm{pN}^{-}$, the probability of survival of $\mathrm{pN}^{-}$patients with UCB: low expression (solid line), $\mathrm{n}=95$; high expression (dashed line), $\mathrm{n}=74$. (I) $\mathrm{pN}$, probability of survival of $\mathrm{pN}^{+}$patients with $U C B$ : low expression (solid line), $\mathrm{n}=3$; high expression (dashed line), $\mathrm{n}=43$.

Abbreviations: PKM2, pyruvate kinase M2; UCB, urothelial carcinoma of the bladder.

Table 3 Multivariate analysis of overall patient survival (Cox regression model)

\begin{tabular}{|c|c|c|c|}
\hline Characteristics & HR & $95 \% \mathrm{Cl}$ & $P$-value \\
\hline PKM2 expression (low vs high) & 3.197 & $1.682-6.076$ & $<0.001$ \\
\hline Histological grade ( $\mathrm{Gl}$ vs $\mathrm{G} 2$ vs $\mathrm{G} 3$ ) & 2.446 & $1.67 \mid-3.579$ & $<0.001$ \\
\hline $\begin{array}{l}\mathrm{PT} \text { classification ( } \mathrm{PTa} / \mathrm{p} T \text { is vs } \mathrm{p} T \mathrm{I} \\
\text { vs } \mathrm{p} T 2-4)\end{array}$ & 2.867 & $1.90 \mathrm{I}-4.323$ & $<0.001$ \\
\hline $\mathrm{pN}$ classification $\left(\mathrm{pN}^{-}\right.$vs $\left.\mathrm{pN}^{+}\right)$ & 2.013 & $1.236-3.279$ & 0.005 \\
\hline Tumor size $(\mathrm{cm})(\leq 3$ vs $>3)$ & 1.235 & $0.728-1.692$ & 0.331 \\
\hline
\end{tabular}

Abbreviations: $\mathrm{HR}$, hazard ratio; $\mathrm{Cl}$, confidence interval; PKM2, pyruvate kinase $\mathrm{M} 2$.
Previous studies have demonstrated that PKM2 is a critical oncogene in various human cancers. Papadaki et al analyzed the expression of PKM2 in tumor samples from 148 and 157 non-small-cell lung cancer (NSCLC) patients treated with front-line platinum-based chemotherapy in the training and validation sets, respectively. In addition, 85 NSCLC patients treated with non-platinum-containing regimens were included as a control group. In the validation and training sets, high PKM2 mRNA levels were associated with decreased 
progression-free survival (PFS), OS, and disease control rate (all $P<0.05$ ) compared with patients with low PKM2 levels. No correlation was found between PKM2 mRNA levels and PFS or OS in the control group. Multivariate analysis revealed that high PKM2 mRNA expression was an independent predictive factor of a poor outcome. ${ }^{28}$

Lim et al analyzed PKM2 expression in gastric cancer using gene expression microarrays and tissue microarrays. Their results revealed that PKM2 expression was significantly increased in primary gastric cancers compared with adjacent normal tissues from the same patients. Furthermore, PKM2 expression was associated with shorter OS $(P<0.05) .{ }^{29} \mathrm{Chao}$ et al evaluated PKM2 expression in ovarian cancer, reporting that patients with higher PKM2 levels correlated significantly with malignancy groups $(P<0.001)$ and pathogenesis models $(P<0.001)$ and had poor PFS $(P=0.01)$ compared with patients exhibiting lower PKM2 levels. ${ }^{30}$

In this study, we collected 10 UCB tissues. The expression of PKM2 in UCB tissues was detected by Western blot, qRTPCR, and IHC. The results showed that the protein and mRNA levels of PKM2 in UCBs were significantly higher than in the control group. By analyzing the relationship between PKM2 protein and mRNA expression and clinicopathological features, we found that the expression of PKM2 correlated positively with the clinical stage and tumor grade of UCB patients. Furthermore, patients with high PKM2 expression had a poor prognosis. The results demonstrate that PKM2 is a potentially valuable biomarker that predicts recurrence and survival after surgical resection and that upregulation of PKM2 may provide a selective advantage in UCB tumorigenesis.

Obtaining sufficient energy is essential for cell survival. Cells grow primarily by glycolysis and oxidative phosphorylation, the latter of which is more efficient - in normal tissues, $\sim 90 \%$ of ATP is derived from oxidative phosphorylation vs $10 \%$ from glycolysis. ${ }^{31}$ In the presence of oxygen, most mature differentiated cells mainly metabolize glucose into carbon dioxide by oxidation of glycolytic pyruvate in the mitochondrial tricarboxylic acid cycle, maximizing energy production with little lactic acid. Only in the absence of oxygen is pyruvate converted into lactic acid by lactate dehydrogenase. However, regardless of whether they are under aerobic or anaerobic conditions, tumor cells consume large amounts of glucose and produce significant amounts of lactic acid.

PKM2 can directly or indirectly regulate the proliferation, apoptosis, and metastasis of tumor cells. ${ }^{22}$ PKM2 regulates tumor angiogenesis through two mechanisms: 1) enhancement of endothelial cell proliferation, migration, and cellextracellular matrix adhesion; ${ }^{8,32}$ and 2) a PKM2/NF- $\mathrm{KB} /$ miR-148a/152 feedback loop. ${ }^{33}$ With regard to apoptosis,
PKM2 phosphorylates $\mathrm{Bcl} 2$ to prevent the binding of $\mathrm{Cul} 3-$ based E3 ligase to Bcl2, leading to its degradation and the inhibition of apoptosis. ${ }^{34}$ Concerning tumor metastasis, PKM2 is highly associated with STS-induced epithelialmesenchymal transition, which is related to the migration and invasion of cancer cells. ${ }^{35}$

It has been reported that the concentration of blood PKM2 is associated with the sensitivity of lung cancer to chemotherapy drugs. ${ }^{36}$ The serum level of PKM2 in RCC patients was higher than in the healthy control group, and the serum level of PKM2 correlated with stage and metastasis status in RCC patients. Serum PKM2 levels decreased to normal after radical nephrectomy, whereas in cases of recurrence or metastasis, they rose again, indicating that serum PKM2 level is a biomarker for surveillance, recurrence, and metastasis in RCC. ${ }^{26,37}$ Therefore, PKM2 measurement is a useful tool for the diagnosis of cancer and may be used as a diagnostic marker for tumors and a marker of drug efficacy and tumor recurrence in RCC. We determined PKM2 protein and mRNA levels in $\mathrm{BCa}$ tissues and found that this marker was associated with TNM staging and pathological grading. Thus, PKM2 may serve as a biomarker for BCa and contribute to its progressive features and poor prognosis.

Overall, this study has several limitations, including its retrospective design and the small cohort of cases from a single center. Furthermore, because of the complicated mechanism of UCB, more studies are needed to elucidate the specific mechanism of PKM2 in UCB.

\section{Conclusion}

The expression of PKM2 protein and mRNA is increased significantly in UCB and correlates positively with histological grade and TNM staging. PKM2 may be involved in $\mathrm{BCa}$ growth and metastasis and may serve as a tumor marker for the degree of malignancy of $\mathrm{BCa}$.

\section{Acknowledgment}

This work was supported by the Youth Research Project of Fujian Provincial Health and Family Planning Commission (2015-2-47), Project of Science and Technology Bureau of Xiamen (3502Z20154022), and the Natural Science Foundation of Fujian Province (2060203).

\section{Disclosure}

The authors report no conflicts of interest in this work.

\section{References}

1. Torre LA, Bray F, Siegel RL, Ferlay J, Lortet-Tieulent J, Jemal A. Global cancer statistics, 2012. CA Cancer J Clin. 2015;65(2):87-108. 
2. Reinacher M, Eigenbrodt E. Immunohistological demonstration of the same type of pyruvate kinase isoenzyme (M2-Pk) in tumors of chicken and rat. Virchows Arch B Cell Pathol Incl Mol Pathol. 1981;37(1): $79-88$.

3. Eigenbrodt E, Reinacher M, Scheefers-Borchel U, Scheefers H, Friis R. Double role for pyruvate kinase type M2 in the expansion of phosphometabolite pools found in tumor cells. Crit Rev Oncog. 1992; 3(1-2):91-115.

4. Gumińska M, Ignacak J, Kedryna T, Stachurska MB. Tumor-specific pyruvate kinase isoenzyme M2 involved in biochemical strategy of energy generation in neoplastic cells. Acta Biochim Pol. 1997;44(4): 711-724.

5. Hacker HJ, Steinberg P, Bannasch P. Pyruvate kinase isoenzyme shift from L-type to M2-type is a late event in hepatocarcinogenesis induced in rats by a choline-deficient/DL-ethionine-supplemented diet. Carcinogenesis. 1998;19(1):99-107.

6. Steinberg P, Klingelhöffer A, Schäfer A, et al. Expression of pyruvate kinase M2 in preneoplastic hepatic foci of N-nitrosomorpholine-treated rats. Virchows Arch. 1999;434(3):213-220.

7. Eigenbrodt E, Gerbracht U, Mazurek S, Presek P, Friis R. Carbohydrate metabolism and neoplasia: new perspectives for diagnosis and therapy. In: Pretlow TG, Pretlow TP, editors. Biochemical and Molecular Aspects of Selected Cancers. Vol 2. San Diego, CA: Academic Press; 1994: 311-381.

8. Li Z, Yang P, Li Z. The multifaceted regulation and functions of PKM2 in tumor progression. Biochim Biophys Acta. 2014;1846(2):285-296.

9. Staal GEJ, Rijksen G. Pyruvate kinase in selected human tumors. In: Pretlow TG, Prelow TP, editors. Biochemical and Molecular Aspects of Selected Cancers. Vol 2. San Diego, CA: Academic Press; 1991:313-337.

10. Noguchi T, Inoue H, Tanaka T. The M1- and M2-type isozymes of rat pyruvate kinase are produced from the same gene by alternative RNA splicing. J Biol Chem. 1986;261(29):13807-13812.

11. Varga Z, Hegele A, Stief T, Heidenreich A, Hofmann R. Determination of pyruvate kinase type tumor M2 in human renal cell carcinoma: a suitable tumor marker? Urol Res. 2002;30(2):122-125.

12. Weinberger R, Appel B, Stein A, Metz Y, Neheman A, Barak M. The pyruvate kinase isoenzyme M2 (Tu M2-PK) as a tumour marker for renal cell carcinoma. Eur J Cancer Care (Engl). 2007;16(4):333-337.

13. Ahmed AS, Dew T, Lawton FG, et al. M2-PK as a novel marker in ovarian cancer. A prospective cohort study. Eur J Gynaecol Oncol. 2007;28(2): 83-88.

14. Ahmed AS, Dew T, Lawton FG, et al. Tumour M2-PK as a predictor of surgical outcome in ovarian cancer, a prospective cohort study. Eur J Gynaecol Oncol. 2007;28(2):103-108.

15. Lüftner D, Mesterharm J, Akrivakis C, et al. Tumor type M2 pyruvate kinase expression in advanced breast cancer. Anticancer Res. 2000; 20(6D):5077-5082.

16. Huang L, Yu Z, Zhang Z, Ma W, Song S, Huang G. Interaction with pyruvate kinase M2 destabilizes tristetraprolin by proteasome degradation and regulates cell proliferation in breast cancer. Sci Rep. 2016;6:22449.

17. Schneider J, Neu K, Grimm H, Velcovsky HG, Weisse G, Eigenbrodt E. Tumor M2-pyruvate kinase in lung cancer patients: immunohistochemical detection and disease monitoring. Anticancer Res. 2002; 22(1A):311-318.

18. Sobin LH, Gospodariwicz M, Wittekind C, editors. TNM Classification of Malignant Tumors, 7th Edition. Hoboken, NJ: Wiley-Blackwell; 2009.

OncoTargets and Therapy

\section{Publish your work in this journal}

OncoTargets and Therapy is an international, peer-reviewed, open access journal focusing on the pathological basis of all cancers, potential targets for therapy and treatment protocols employed to improve the management of cancer patients. The journal also focuses on the impact of management programs and new therapeutic agents and protocols on
19. Wu D, Ding Y, Wang S, Zhang Q, Liu L. Increased expression of high mobility group box 1 (HMGB1) is associated with progression and poor prognosis in human nasopharyngeal carcinoma. J Pathol. 2008;216(2): $167-175$.

20. Tamada M, Suematsu M, Saya H. Pyruvate kinase M2: multiple faces for conferring benefits on cancer cells. Clin Cancer Res. 2012;18(20): $5554-5561$

21. Warburg O. On the origin of cancer cells. Science. 1956;123(3191): 309-314.

22. He X, Du S, Lei T, et al. PKM2 in carcinogenesis and oncotherapy. Oncotarget. 2017;8(66):110656-110670.

23. Iqbal MA, Gupta V, Gopinath P, Mazurek S, Bamezai RN. Pyruvate kinase M2 and cancer: an updated assessment. FEBS Lett. 2014;588(16): 2685-2692.

24. Mazurek S, Boschek CB, Hugo F, Eigenbrodt E. Pyruvate kinase type M2 and its role in tumor growth and spreading. Semin Cancer Biol. 2005; 15(4):300-308

25. Christofk HR, Vander Heiden MG, Harris MH, et al. The M2 splice isoform of pyruvate kinase is important for cancer metabolism and tumour growth. Nature. 2008;452(7184):230-233.

26. Wechsel HW, Petri E, Bichler KH, Feil G. Marker for renal cell carcinoma (RCC): the dimeric form of pyruvate kinase type M2 (Tu M2-PK). Anticancer Res. 1999;19(4A):2583-2590.

27. Bluemlein K, Grüning NM, Feichtinger RG, Lehrach H, Kofler B, Ralser M. No evidence for a shift in pyruvate kinase PKM1 to PKM2 expression during tumorigenesis. Oncotarget. 2011;2(5):393-400.

28. Papadaki C, Sfakianaki M, Lagoudaki E, et al. PKM2 as a biomarker for chemosensitivity to front-line platinum-based chemotherapy in patients with metastatic non-small-cell lung cancer. Br J Cancer. 2014; 111(9):1757-1764.

29. Lim JY, Yoon SO, Seol SY, et al. Overexpression of the M2 isoform of pyruvate kinase is an adverse prognostic factor for signet ring cell gastric cancer. World J Gastroenterol. 2012;18(30):4037-4043.

30. Chao TK, Huang TS, Liao YP, et al. Pyruvate kinase M2 is a poor prognostic marker of and a therapeutic target in ovarian cancer. PLoS One. 2017;12(7):e0182166

31. Gillies RJ, Gatenby RA. Hypoxia and adaptive landscapes in the evolution of carcinogenesis. Cancer Metastasis Rev. 2007;26(2):311-317.

32. Li L, Zhang Y, Qiao J, Yang JJ, Liu ZR. Pyruvate kinase M2 in blood circulation facilitates tumor growth by promoting angiogenesis. $J$ Biol Chem. 2014;289(37):25812-25821.

33. Xu Q, Liu LZ, Yin Y, et al. Regulatory circuit of PKM2/NF-kB/ $\mathrm{miR}-148 \mathrm{a} / 152$-modulated tumor angiogenesis and cancer progression. Oncogene. 2015;34(43):5482-5493.

34. Liang J, Cao R, Wang X, et al. Mitochondrial PKM2 regulates oxidative stress-induced apoptosis by stabilizing Bcl2. Cell Res. 2017;27(3): 329-351.

35. Hamabe A, Konno M, Tanuma N, et al. Role of pyruvate kinase M2 in transcriptional regulation leading to epithelial-mesenchymal transition. Proc Natl Acad Sci U S A. 2014;111(43):15526-15531.

36. Schneider J, Neu K, Velcovsky HG, Morr H, Eigenbrodt E. Tumor $\mathrm{M} 2$-pyruvate kinase in the follow-up of inoperable lung cancer patients: a pilot study. Cancer Lett. 2003;193(1):91-98.

37. Roigas J, Deger S, Schroeder J, et al. Tumor type M2 pyruvate kinase expression in metastatic renal cell carcinoma. Urol Res. 2003;31(6): $358-362$.

patient perspectives such as quality of life, adherence and satisfaction The manuscript management system is completely online and includes a very quick and fair peer-review system, which is all easy to use. Visit http://www.dovepress.com/testimonials.php to read real quotes from published authors. 